\title{
Human umbilical cord matrix-derived stem cells expressing interferon- $\beta$ gene inhibit breast cancer cells via apoptosis
}

\author{
Ching-Ju Shen ${ }^{1,2}$, Te-Fu Chan ${ }^{2}$, Chien-Chung Chen ${ }^{3}$, Yi-Chiang Hsu ${ }^{4,5}$, Cheng-Yu \\ Long $^{2}$, Chung-Sheng Lai ${ }^{1,6}$ \\ ${ }^{1}$ Graduate Institute of Medicine, College of Medicine, Kaohsiung Medical University, Kaohsiung, Taiwan \\ ${ }^{2}$ Department of Obstetrics and Gynecology, Kaohsiung Medical University Hospital, Kaohsiung Medical University, Kaohsiung, \\ Taiwan \\ ${ }^{3}$ Department of Plastic and Reconstruction Surgery, E-Da Hospital, Taiwan \\ ${ }^{4}$ Graduate Institute of Medical Science, College of Health Sciences, Chang Jung Christian University, Tainan, Taiwan \\ ${ }^{5}$ Innovative Research Center of Medicine, College of Health Sciences, Chang Jung Christian University, Tainan, Taiwan \\ ${ }^{6}$ Division of Plastic Surgery, Department of Surgery, Kaohsiung Medical University Hospital, Kaohsiung Medical University, \\ Kaohsiung, Taiwan
}

Correspondence to: Chung-Sheng Lai, email: chshla@kmu.edu.tw

Keywords: breast carcinoma, MDA-MB-231 cells, Hs578T cells, interferon- $\beta$, stem cell therapy

Received: November 04, 2015

Accepted: March 14, 2016

Published: April 26, 2016

ABSTRACT

Human umbilical cord mesenchymal stem cells (hUCMSCs) derived from the umbilical cord matrix have been reported to be used as anti-tumor gene carrier for attenuation of tumor growth, which extends the half-life and lowers the unexpected cytotoxicity of the gene in vivo. Interferon- $\beta$ (IFN $\beta$ ) is known to possess robust antitumor effects on different types of cancer cell lines in vitro. The present study was aimed to investigate the anti-tumor effect of IFN $\beta$ gene-transfected hUCMSCs (IFN $\beta$ hUCMSCs) on breast cancer cells with emphasis on triple negative breast carcinoma. Our findings revealed that the co-culture of IFN $\beta$-hUCMSCs with the human triple negative breast carcinoma cell lines MDA-MB-231 or Hs578T significantly inhibited growth of both carcinoma cells. In addition, the culture medium conditioned by these cells also significantly suppressed the growth and induced apoptosis of both carcinoma cells. Further investigation showed that the suppressed growth and the apoptosis induced by co-culture of IFN $\beta$-hUCMSCs or conditioned medium were abolished by pretreating anti-IFN $\beta$ neutralizing antibody. These findings indicate that IFN $\beta-$ hUCMSCs triggered cell death of breast carcinoma cells through IFN- $\beta$ production, thereby induced apoptosis and suppressed tumor cell growth. In conclusion, we demonstrated that IFN $\beta$-hUCMSCs inhibited the growth of breast cancer cells through apoptosis. With potent anti-cancer activity, it represents as an anti-cancer cytotherapeutic modality against breast cancer.

\section{INTRODUCTION}

Breast cancer is the commonest malignancy in most countries in Asia, and the incidence is increasing at a more rapid rate than in western countries, which may be due to changes in the lifestyle and diet $[1,2]$. The most major risk factors for breast cancer are known to include hormone replacement therapy such as prolonged exposure to estrogen and/or progesterone and reproductive history [3]. Besides, the importance of estrogen in breast cancer development is also supported by studies demonstrating the occurrence of great changes in estrogen signaling and in the expression of estrogen receptors (ERs), ER alpha and ER beta, during breast carcinogenesis and progression [4-6]. Nowadays, surgical resection followed by radiotherapy and/ or chemotherapy is still the most common treatments for breast cancer to prevent its metastasis and recurrence [7]. Therefore, novel treatment strategies for breast cancer are needed.

Interferon- $\beta$ (IFN $\beta$ ) has been reported to exhibit robustly inhibitory effects on tumor cell growth and induce apoptosis in vitro [8-10]. However, IFN $\beta$ gene therapy has 
not been successfully used in in vivo because of its short half-life, as well as the maximally tolerated dose is lower than the effective dose. Although viral vector-based gene delivery is not cancer tissue-specific, several studies have demonstrated that IFN $\beta$ gene therapy using adenoviral vectors is effective in several cancers such as ovarian cancer [11], bladder cancer [12], glioma [13], and lung cancer [14]. In addition, the effectiveness of adenoviral vector-based gene delivery to tumor tissues remains unclear. Actually, intratumoral injection of virus vectors showed restricted target protein expression in the cells adjacent to the injection site [15]. To conquer the problem, human bone marrowderived mesenchymal stem cells (MSCs) have been utilized as biological vehicles for IFN $\beta$ gene delivery. This MSCbased IFN $\beta$ therapy using systemic administration has been shown to be effective in suppressing metastasis of breast cancer, melanoma [16] and glioma [17, 18].

MSCs derived from the human umbilical cord matrix (hUCMSCs), human postnatal stem cells, possess beneficial properties for therapeutic uses including relatively large cell number of harvest, propagated without any feeder cells, and stored after birth without significant risks to the donor. Rachakatla et al. also reported that no teratomas were formed in the SCID mice with injection of hUCMSCs [19]. In addition systemically administered IFN $\beta$ gene transfected hUCMSCs (IFN $\beta$-hUCMSCs) is capable of migrating to tumor sites and attenuating growth of breast tumor metastasized to breast [19]. These findings indicate that hUCMSCs can be potential biological vehicles for tumortargeted delivery of therapeutic agents or genes. However, since this novel therapy has never been applied to the most difficult cancers such as triple negative breast cancer, the aim of this study was to evaluate the therapeutic potential of the IFN $\beta$-hUCMSCs for treating triple negative breast carcinomas. Our findings demonstrated that hUCMSCs producing IFN $\beta$ were capable of inhibiting growth of human triple negative breast cancer cells via both extrinsic and intrinsic apoptotic pathways.

\section{RESULTS}

\section{IFN $\beta$-hUCMSCs significantly inhibited cell growth of MDA-MB-231 and Hs578T cells}

To evaluate the effect of IFN $\beta$-hUCMSCs on cancer cell proliferation, MDA-MB-231 and Hs578T human triple negative breast cancer cells were individually cultured in the bottom of Transwell culture dishes and either hUCMSCs or IFN $\beta$-hUCMSCs were co-cultured in the inserts. After $72 \mathrm{~h}$ incubation, numbers of live cells in the bottom of culture dishes were counted. The results revealed that live cell numbers of both MDA-MB-231 and Hs578T cells were significantly decreased after the co-culture with IFN $\beta$-hUCMSCs (Figure 1). In parallel, live cell numbers in both cell lines co-culture with vehicle hUCMSCs were also decreased; however, the decrease of live cell numbers of the tumor cells co-culture with vehicle hUCMSCs was insignificant as compared to tumor cells alone.

\section{IFN $\beta$ in the conditioned medium derived from IFN $\beta$-hUCMSCs reduced cell growth of MDA- MB-231 and Hs578T}

Since co-culture with IFN $\beta$-hUCMSCs reduced growth of breast cancer cells, the role of IFN $\beta$ in the reduced cell growth was further investigated. As shown

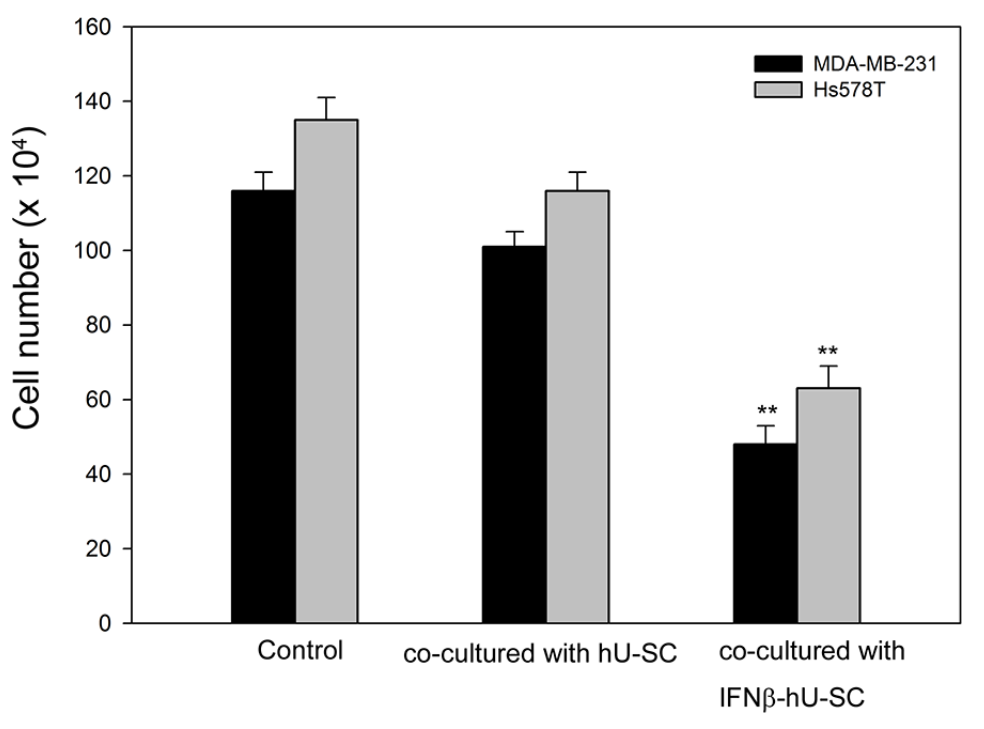

Figure 1: Effect of hUCMSCs and IFN $\beta$-hUCMSCs co-culture on growth of MDA-MB-231 and Hs578T. Cells $\left(3 \times 10^{5}\right.$ cells/well) were seeded in 6-well plates and allowed to attach to culture dishes. hUCMSCs or IFN $\beta$-hUCMSCs $\left(3 \times 10^{5}\right.$ cells $)$ were seeded in the cell culture inserts $(3.0 \mu \mathrm{m}$ pore size). The cells were cultured with hUCMSCs growth medium for $72 \mathrm{~h}$, and the cell number was counted using a hemocytometer. Data were presented as means $\pm \mathrm{SD}$ of the three independent experiments. $* * P<0.01$ as compared to negative control. 
in Figure 2, incubation with medium conditioned with IFN $\beta$-hUCMSCs significantly suppressed growth of both MDA-MB-231 and Hs578T cell lines, and the cell growth was lowered down to $53.6 \pm 3.7 \%$ and $51.1 \pm 6.3 \%$ of control, respectively. Besides, pre-addition of anti-human IFN $\beta$ monoclonal antibody to the conditioned medium neutralized the suppression of cell growth by the IFN $\beta$ hUCMSCs conditioned medium (Figure 2). In parallel, although incubation with medium conditioned with vehicle hU-SC appeared to reduce live cell number of both tumor cell lines, the decrease of the live cell number was insignificant as compared to incubation with control medium. In addition, the pre-addition of anti-human IFN $\beta$ monoclonal antibody to the conditioned medium also revealed the similar effects on both tumor cell lines as the conditioned medium alone. Collectively, these observations showed that IFN $\beta$ released in IFN $\beta$-hUCMSCs conditioned medium suppressed the growth of both breast tumor cells

\section{Medium conditioned with IFN $\beta$-hUCMSCs induced apoptosis of MDA-MB-231 and Hs578T cells}

To investigate whether apoptosis was involved in the inhibited cell growth by IFN $\beta$-hUCMSCs conditioned medium, flow cytometric analysis using PI-Annexin V staining was performed. As shown in Figure 3, culture with the conditioned medium derived from IFN $\beta$-hUCMSCs for $24 \mathrm{~h}$ significantly increased percentage of apoptotic cells (early and late apoptosis) for both MDA-MB-231 and $\mathrm{H} 358$ cells up to $41.5 \pm 3.2 \%$ and $43.6 \pm 4.1 \%$, respectively. In addition, apoptosis was observed as early as in $6 \mathrm{~h}$ after incubation with the conditioned medium (data not shown).

The apoptosis induced by IFN $\beta$-hUCMSCs conditioned medium was also evaluated by immunoblotting analysis of caspase cascades. Consistent with flow cytometry analysis, the protein levels of cleaved caspase-8, cleaved caspase- 9 and cleaved caspase- 3 were increased in response to IFN $\beta$-hUCMSCs conditioned medium (Figure 4). Furthermore, culture with IFN $\beta$-hUCMSCs conditioned medium contributed to decrease of antiapoptotic Bcl-2 level (Figure 4). Together, these results showed that IFN $\beta$-hUCMSCs conditioned medium significantly induced cell apoptosis and triggered activation of caspase cascades in the breast tumor cells.

\section{Involvement of JAK/STAT signaling in medium conditioned with IFN $\beta$-hUCMSCs induced apoptosis of MDA-MB-231}

JAK/STAT signaling is known to play an important role in type I interferon induced apoptosis. Therefore, the involvement of JAK/STAT signaling in IFN $\beta$ - hUCMSCs induced apoptosis was then investigated. As shown in Figure 5, culture with the conditioned medium derived from IFN $\beta$ - hUCMSCs for $24 \mathrm{~h}$ significantly increased phosphorylation of STAT-1 (pSTAT-1) in MDA-MB-231 cells. In addition, inhibition of JAK reduced both pSTAT-1 and cleavage of procaspase-3. These results revealed that JAK/STAT signaling involved in IFN $\beta$ - hUCMSCs induced apoptosis.

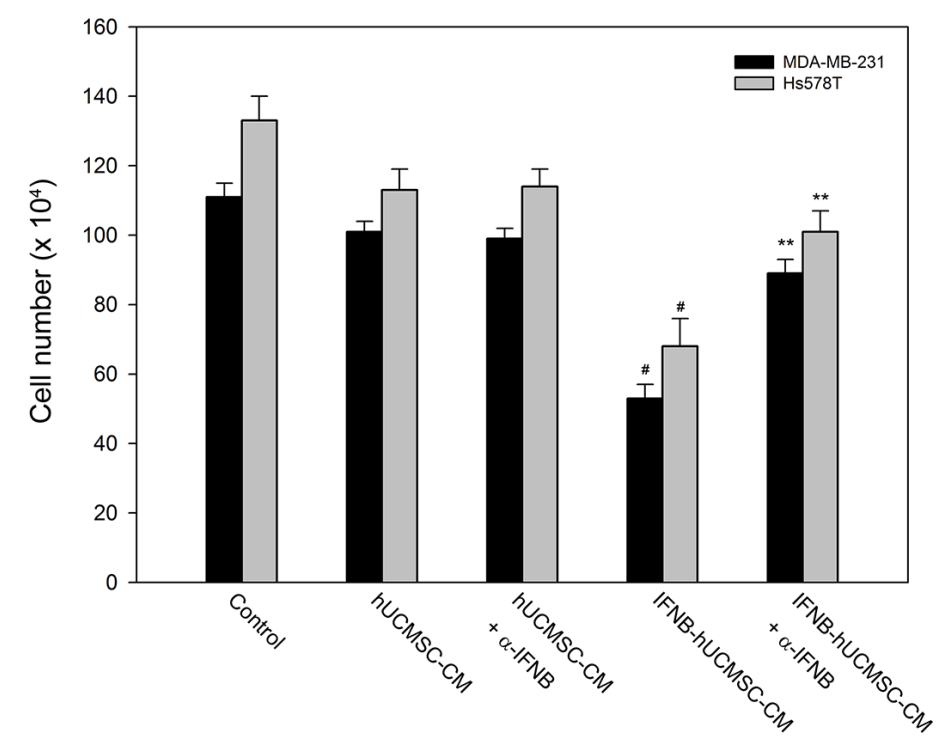

Figure 2: Effect of hUCMSCs or IFN $\beta$-hUCMSCs conditioned medium with or without IFN $\beta$ neutralizing antibody on growth of MDA-MB-231 and Hs578T. Cells $\left(3 \times 10^{5}\right.$ cells/well $)$ were seeded in 6-well plates and allowed to attach for $24 \mathrm{~h}$. After removing culture medium, the cells were incubated with the hUCMSCs or IFN $\beta$-hUCMSCs conditioned medium with or without IFN $\beta$ neutralizing antibody at $5.0 \mu \mathrm{g} / \mathrm{mL}$ for $72 \mathrm{~h}$, and then the cell number was counted using a hemocytometer. Data were presented as means \pm $\mathrm{SD}$ of the three independent experiments. ${ }^{* *} P<0.01$ as compared to negative control. ${ }^{\#} P<0.05$ as compared to IFN $\beta$-hU-SC conditioned medium. 

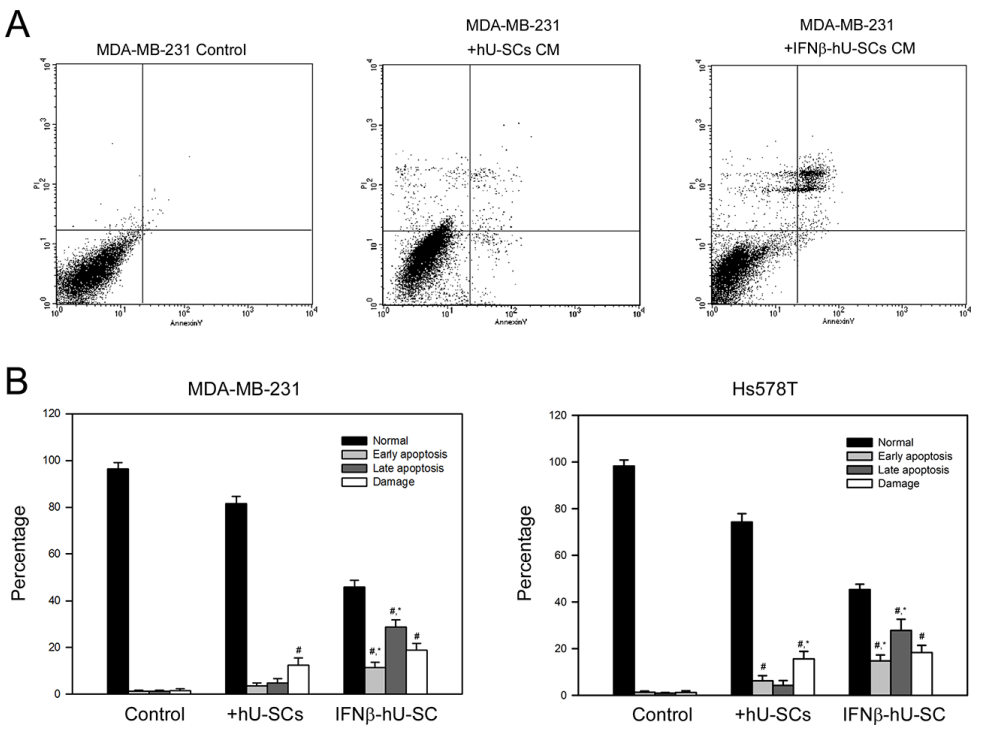

Figure 3: Effect of hUCMSCs or IFN $\beta$-hUCMSCs conditioned medium on the induction of apoptosis in MDA-MB-231 and Hs578T. Cells $\left(3 \times 10^{5}\right.$ cells/well $)$ were seeded in 6-well plates and allowed to attach for $24 \mathrm{~h}$. After removing culture medium, the cells were incubated with the hUCMSCs or IFN $\beta$-hUCMSCs conditioned medium for $24 \mathrm{~h}$. The cells were collected, fixed and reacted with PI and Annexin V-FITC, and then subjected to flow cytometric analysis. (A) Representative flow cytometric analysis. (B) Quantitation for the flow cytometric analysis. PI/Annexin-FITC: -/-, normal; PI/Annexin-FITC: -/+, early apoptosis; PI/Annexin-FITC: +/+, late apoptotic (late); PI/Annexin-FITC $=+/-$, damaged cells. Data were presented as means $\pm \mathrm{SD}$ of the three independent experiments. ${ }^{\sharp} P<0.05$ as compared to control. ${ }^{*} P<0.05$ as compared to hUCMSCs conditioned medium.
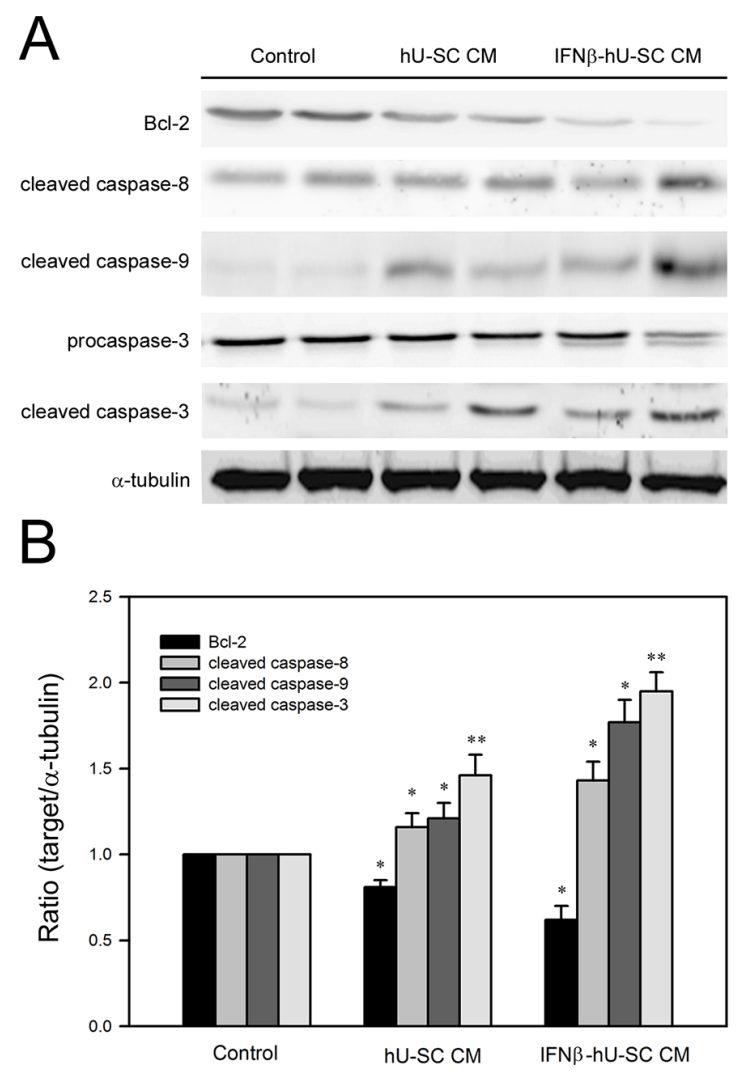

Figure 4: Effect of hUCMSCs or IFN $\beta$-hUCMSCs conditioned medium on caspase cascades in MDA-MB-231 and Hs578T. Cells $\left(1 \times 10^{6}\right.$ cells) were seeded in culture plates and allowed to attach for $24 \mathrm{~h}$. After removing culture medium, the cells were incubated with the hUCMSCs or IFN $\beta$-hUCMSCs conditioned medium for $48 \mathrm{~h}$. (A) The cells were collected and lysed for immunodetection of Bcl-2 and caspases using specific antibodies. (B) Quantitative data were acquired using densitometric analysis from three independent experiments. ${ }^{*}$ and ${ }^{* *} p<0.05$ and $p<0.01$ as compared to negative control. 


\section{DISCUSSION}

Mounting evidences suggest that endogenous apoptosis inducers and cell growth regulators are important targets for effective cancer therapy [23-25]. Recently, a number of such gene products and inhibitors for growth factors are increasingly tested in clinical trials [26, 27]. IFN- $\beta$ is a cytokine that robustly induces caspase-mediated apoptosis of cancer cells through activating different pathways such as mitochondrial and Jak-Stat signaling $[28,29]$. IFN- $\beta$ is also a potent inhibitor of proliferation of many cancer cell lines in vitro [30]. However, use of IFN $\beta$ for cancer therapy is difficult due to its short halflife, as well as due to the low systemic tolerated dose that is inefficient to exert the apoptosis-inducing effects $[31,32]$. Studeny et al. [16] has shown that the local production of IFN $\beta$ in tumor tissues plays an important role in IFN $\beta$-based cancer therapy. Thus, stem cell-based therapeutic gene delivery to tumor tissues appears to be a promising approach for local production of therapeutic cytokine and avoiding the adverse effects of systemic cytotoxic cytokines. Similarly, our present study indicates that hUCMSCs based IFN $\beta$ gene delivery effectively suppresses growth of triple negative breast cancer cells, which is one of the most difficult cancers to treat. Further studies in vivo are required to demonstrate the homing property of IFN $\beta$-hUCMSCs to breast tumor tissue.

Aberrant cells such as mutated or proliferating neoplastic cells are removed by programmed cell death, namely apoptosis [33]. Two well-known pathways, extrinsic and intrinsic pathways, are responsible for triggering apoptosis [34]. For the growth inhibition by IFN $\beta$, our results revealed that IFN $\beta$-hUCMSCs conditioned medium significantly increased Annexin V-FITC positive cells and the increase of Annexin V-FITC positive cells was diminished by pretreating with IFN $\beta$ neutralizing antibody, indicating IFN $\beta$ production plays a central role in the induction of apoptosis. Moreover, these findings also demonstrate that IFN $\beta$-hUCMSCs $\mathrm{C}$ is an efficient gene delivery vector that produces enough IFN $\beta$ to induce apoptosis and the consequent cell growth inhibition. Consistent to increase of Annexin V-FITC positive cells, the immunoblotting analysis showed that IFN $\beta$-hUCMSCs conditioned medium significantly triggered activation of caspase-8, caspase- 9 and caspase- 3 . Taken together, these findings indicate that the IFN $\beta$-dependent cell growth attenuation attributes to the activation of both extrinsic and intrinsic apoptosis pathways. To the best of our knowledge, this is the first demonstration of the efficacy of IFN $\beta$ gene transfected stem cell therapy on breast carcinomas, proposing that IFN $\beta$-hUCMSCs is a potential therapeutic agent for breast cancer. Nevertheless, the effective dose of IFN $\beta$-hUCMSCs as well as working concentration of IFN- $\beta$ in preclinical and clinical setting requires further studies.

Regarding tumor tissue-targeted homing of hUCMSCs, interactions between chemokines produced by tumor tissues and receptor expression in stem cells have been suggested. Various chemokines can be secreted by tumors, including vascular endothelial growth factor (VEGF), Transforming growth factor (TGF) family members, fibroblast growth factor (FGF) family members, Platelet-derived growth factor (PDGF) family members, MCP-1, EGF, and IL-8 [35]. Interestingly, the bone marrow MSCs exhibit a tropism for damaged or rapidly growing tissues as well as tumors [36, 37]. Previous study also indicates that hUCMSCs home to tumor tissues but not to healthy tissues and express multiple chemokine receptors, such as SDFR1, TGFBR3, FGFR2 [19, 38]. Thus, it is conceivable that IFN $\beta$-hUCMSCs administered via the tail vein exhibited a selective from the tumor and/or tumor-

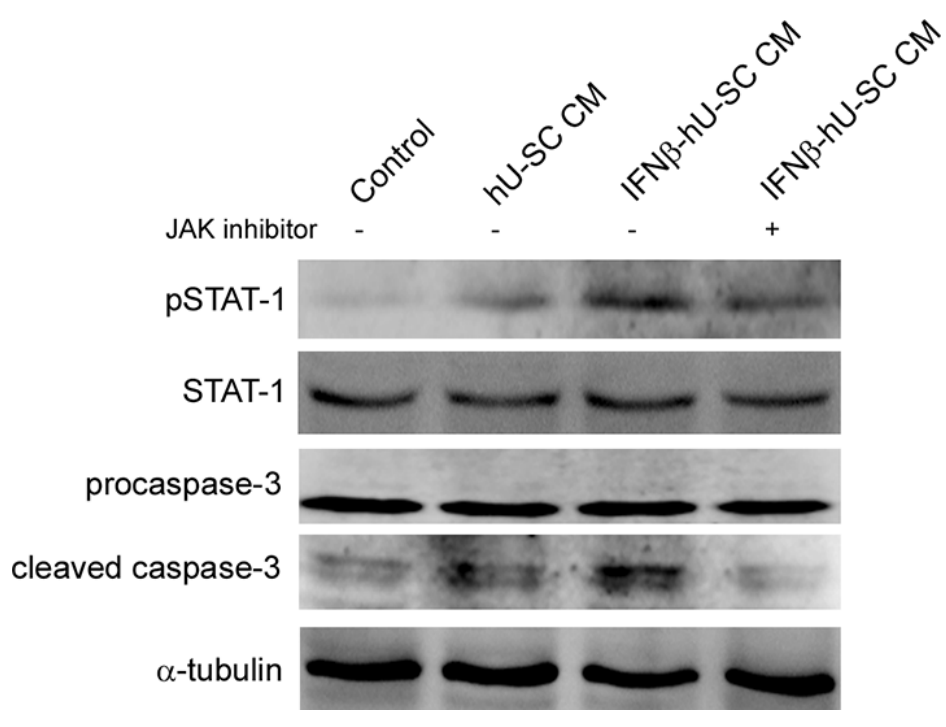

Figure 5: Involvement of Jak-Stat pathway in IFN $\boldsymbol{\beta}$-hUCMSCs conditioned medium induced apoptosis. MDA-MB-231 cells were pretreated with JAK inhibitor $(5 \mu \mathrm{M})$ for $2 \mathrm{~h}$ and then treated with indicated conditioned medium for $48 \mathrm{~h}$. The treated cells were collected and lysed for immunodetection of pSTAT-1, STAT-1 and caspase-3. $\alpha$-tubulin was used as internal control. 
associated cells. These properties of hUCMSCs encourage their development as a therapeutic agent for tumors.

\section{MATERIALS AND METHODS}

\section{Reagents}

RPMI-1640, L-15 medium, fetal bovine serum (FBS), low glucose Dulbecco's modified Eagle's medium (DMEM), insulin-transferrin-selenium-X (ITS-X), and ALBUMax1 were purchased from Invitrogen (Carlsbad, CA, USA). MCBD 201 medium, ascorbic acid 2-phosphate, and dexamethasone were from Sigma-Aldrich (St. Louis, MO, USA). Epidermal growth factor (EGF) and platelet derived growth factor-BB (PDGF-BB) were from R \& D Systems (Minneapolis, MN, USA).

\section{Cell culture}

The breast cancer cell line MDA-MB-231 and Hs578T was obtained from American Type Culture Collection (ATCC; Rockville, MD, USA) and maintained in DMEM supplemented with $10 \% \mathrm{v} / \mathrm{v}$ fetal bovine serum, $1 \%$ nonessential amino acid, 1\% L-glutamine (Gibco BRL, Gaithersburg, MD, USA) and $100 \mu \mathrm{g} / \mathrm{mL}$ penicillin/streptomycin (Sigma) at $37^{\circ} \mathrm{C}$ in a humidified atmosphere with $5 \% \mathrm{CO}_{2}$. hUCMSCs were prepared from human umbilical cord Wharton's jelly obtained from Taipei Medical University with Institutional Review Board guidance. Culture of hUCMSCs was performed as previously described [19]. The culture medium for hUSC was low glucose DMEM containing 37\% MCDB 201, 2\% FBS, 1\% ITS-X, $1.5 \mathrm{~g} / \mathrm{mL}$ ALBUMax 1, $10 \mathrm{nM}$ dexamethasone, $50 \mu \mathrm{M}$ ascorbic acid 2-phosphate, $1 \mathrm{ng} /$ $\mathrm{mL}$ EGF, $10 \mathrm{ng} / \mathrm{mL}$ PDGF-BB, 100 units $/ \mathrm{mL}$ penicillin and $100 \mu \mathrm{g} / \mathrm{mL}$ streptomycin. The cells were incubated in $5 \%$ $\mathrm{CO}_{2}$ humidified air at $37^{\circ} \mathrm{C}$.

\section{Gene transduction with adenoviral vectors}

The RGD fiber-modified adenoviral vectors encoding genes for human IFN $\beta$ were prepared as previously described [20,21], and the gene transduction to hUCMSCs was performed according to the previous procedure [19]. Twenty four hours (h) after gene transduction, the IFN $\beta$ hUCMSCs were used for the following experiments.

\section{Co-culture of hUCMSCs and IFN $\beta$-hUCMSCs with breast cancer cells}

MDA-MB-231 or Hs578T were maintained in normal culture media at $4 \times 10^{5}$ cells per well in 6 -well plates. Prior to experiments, $3 \times 10^{5} \mathrm{hUCMSCs}$ or IFN $\beta$ hUCMSCs were seeded into Transwell cell culture inserts (3.0 $\mu \mathrm{m}$ pore size, BD Biosciences, San Jose, CA, USA) and cultured with the growth medium for hUCMSCs for
$72 \mathrm{~h}$. The cancer cell cultures were lifted, and the number of the cancer cells was counted using a hemocytometer.

\section{Culture with hUCMSCs and IFN $\beta$-hUCMSCs conditioned media}

The normal culture media were conditioned by culturing IFN $\beta$-hUCMSCs or hUCMSCs for $24 \mathrm{~h}$. The conditioned media were used for treatment of the breast cancer cell lines. Neutralizing antibody against IFN $\beta$ (mouse anti-human IFN $\beta$ monoclonal antibody, Chemicon, Temecula, CA, USA) was added to the conditioned media at a final concentration of $5 \mu \mathrm{g} / \mathrm{mL}$. The viable cancer cell number was counted after $72 \mathrm{~h}$ of culture.

\section{Flow cytometric analysis for apoptotic cells}

The percentage of apoptotic cells was determined using an Annexin V-FITC apoptosis detection kit (Biovision, Mountain View, CA, USA). Briefly, cells were washed with cold PBS and re-suspended in binding buffer (10 mM HEPES, pH 7.4, $140 \mathrm{mM} \mathrm{NaCl}$ and $2.5 \mathrm{mM}$ $\mathrm{CaCl}_{2}$ ) at a concentration of 5-10 $\times 10^{6}$ cells $/ \mathrm{mL}$. Cells were incubated at room temperature with $5 \mu \mathrm{L}$ each of Annexin V-fluorescein isothiocyanate (FITC) and propidium iodide (PI) for 5 minutes, and analyzed on a with a FACS Calibur system (version 2.0, BD Biosciences, Franklin Lakes, NJ, USA) and CellQuest software.

\section{Immunoblotting analysis}

Crude proteins were prepared using lysis buffer (1\% Triton X-100, 0.1\% SDS, $0.25 \mathrm{M}$ sucrose, $1 \mathrm{mM}$ EDTA, $30 \mathrm{mM}$ Tris- $\mathrm{HCl}, \mathrm{pH}$ 8.0) supplemented with protease inhibitor cocktail (Boehringer Mannheim, Indianapolis, IN), and subjected to a $12.5 \%$ SDS-polyacrylamide gel and transferred onto a nitrocellulose membrane as previously described [22]. The blot was subsequently incubated with $5 \%$ nonfat milk in PBS for $1 \mathrm{~h}$, probed with a $1 / 1000$ dilution of primary antibodies against Bcl-2, caspase-3, caspase-8, caspase-9, phospho-STAT-1 (pSTAT-1) and STAT-1 (Cell Signaling Technology, Beverly, MA, USA), or $\alpha$-tubulin $(\alpha-$ TN, Santa Cruz Biotechnology, Santa Cruz, CA, USA) for $2 \mathrm{~h}$, and then reacted with $1 / 2000$ dilution of peroxidase-conjugated secondary antibody for $1 \mathrm{~h}$. All incubations were carried out at $30^{\circ} \mathrm{C}$, and intensive PBS washing was performed between each incubation. After the final PBS wash, the signal was developed by ECL chemiluminescence, and the relative photographic density was quantitated by image analysis system (Fuji Film, Tokyo, Japan).

\section{Statistical analysis}

Data were expressed as means $\pm \mathrm{SD}$ of the three independent experiments. Statistical significance analysis 
was determined by using one-way ANOVA followed by Dunnett for multiple comparisons with the control. The differences were considered significant for $p$ values less than 0.05 .

\section{ACKNOWLEDGMENTS AND FUNDING}

This study was supported by grant from the Kaohsiung Medical University Hospital (No. 8G01 and 7G03).

\section{CONFLICTS OF INTEREST}

The authors declare that there are no conflicts of interest.

\section{REFERENCES}

1. Yip CH. Breast cancer in Asia. Methods Mol Biol. 2009; 471:51-64.

2. Schwingshackl L, Hoffmann G. Adherence to Mediterranean diet and risk of cancer: a systematic review and meta-analysis of observational studies. Int J Cancer. 2014; 135:1884-97.

3. Pike MC, Spicer DV, Dahmoush L, Press MF. Estrogens, progestogens, normal breast cell proliferation, and breast cancer risk. Epidemiol Rev. 1993; 15:17-35.

4. Henderson BE, Feigelson HS. Hormonal carcinogenesis. Carcinogenesis. 2000; 21:427-33.

5. Iwao K, Miyoshi Y, Egawa C, Ikeda N, Noguchi S. Quantitative analysis of estrogen receptor-beta mRNA and its variants in human breast cancers. Int J Cancer. 2000; 88:733-6.

6. Iwao K, Miyoshi Y, Egawa C, Ikeda N, Tsukamoto F, Noguchi S. Quantitative analysis of estrogen receptor-alpha and -beta messenger RNA expression in breast carcinoma by real-time polymerase chain reaction. Cancer. 2000; 89:1732-8.

7. Dickler A, Ivanov O, Francescatti D. Intraoperative radiation therapy in the treatment of early-stage breast cancer utilizing xoft axxent electronic brachytherapy. World J Surg Oncol. 2009; 7:24.

8. Wong VL, Rieman DJ, Aronson L, Dalton BJ, Greig R, Anzano MA. Growth-inhibitory activity of interferon-beta against human colorectal carcinoma cell lines. Int J Cancer. 1989; 43:526-30.

9. Zhang H, Koty PP, Mayotte J, Levitt ML. Induction of multiple programmed cell death pathways by IFN-beta in human non-small-cell lung cancer cell lines. Exp Cell Res. 1999; 247:133-41.

10. Chawla-Sarkar M, Leaman DW, Borden EC. Preferential induction of apoptosis by interferon (IFN)-beta compared with IFN-alpha2: correlation with TRAIL/Apo2L induction in melanoma cell lines. Clin Cancer Res. 2001; 7:1821-31.

11. Xu L, Xie K, Fidler IJ. Therapy of human ovarian cancer by transfection with the murine interferon beta gene: role of macrophage-inducible nitric oxide synthase. Hum Gene Ther. 1998; 9:2699-708.
12. Izawa JI, Sweeney P, Perrotte P, Kedar D, Dong Z, Slaton JW, Karashima T, Inoue K, Benedict WF, Dinney CP. Inhibition of tumorigenicity and metastasis of human bladder cancer growing in athymic mice by interferon-beta gene therapy results partially from various antiangiogenic effects including endothelial cell apoptosis. Clin Cancer Res. 2002; 8:1258-70.

13. Natsume A, Mizuno M, Ryuke Y, Yoshida J. Antitumor effect and cellular immunity activation by murine interferon-beta gene transfer against intracerebral glioma in mouse. Gene Ther. 1999; 6:1626-33.

14. Wilderman MJ, Sun J, Jassar AS, Kapoor V, Khan M, Vachani A, Suzuki E, Kinniry PA, Sterman DH, Kaiser LR, Albelda SM. Intrapulmonary IFN-beta gene therapy using an adenoviral vector is highly effective in a murine orthotopic model of bronchogenic adenocarcinoma of the lung. Cancer Res. 2005; 65:8379-87.

15. L Lang FF, Bruner JM, Fuller GN, Aldape K, Prados MD, Chang S, Berger MS, McDermott MW, Kunwar SM, Junck LR, Chandler W, Zwiebel JA, Kaplan RS, Yung WK. Phase I trial of adenovirus-mediated p53 gene therapy for recurrent glioma: biological and clinical results. J Clin Oncol. 2003; 21:2508-18.

16. Studeny M, Marini FC, Dembinski JL, Zompetta C, Cabreira-Hansen M, Bekele BN, Champlin RE, Andreeff M. Mesenchymal stem cells: potential precursors for tumor stroma and targeted-delivery vehicles for anticancer agents. J Natl Cancer Inst. 2004; 96:1593-603.

17. Studeny M, Marini FC, Champlin RE, Zompetta C, Fidler IJ, Andreeff M. Bone marrow-derived mesenchymal stem cells as vehicles for interferon-beta delivery into tumors. Cancer Res. 2002; 62:3603-8.

18. Nakamizo A, Marini F, Amano T, Khan A, Studeny M, Gumin J, Chen J, Hentschel S, Vecil G, Dembinski J, Andreeff M, Lang FF. Human bone marrow-derived mesenchymal stem cells in the treatment of gliomas. Cancer Res. 2005; 65:3307-18.

19. Rachakatla RS, Marini F, Weiss ML, Tamura M, Troyer D. Development of human umbilical cord matrix stem cellbased gene therapy for experimental lung tumors. Cancer Gene Ther. 2007; 14:828-35.

20. Koizumi N, Mizuguchi H, Utoguchi N, Watanabe Y, Hayakawa T. Generation of fiber-modified adenovirus vectors containing heterologous peptides in both the HI loop and C terminus of the fiber knob. J Gene Med. 2003; 5:267-76.

21. Sengupta S, Ulasov IV, Thaci B, Ahmed AU, Lesniak MS. Enhanced transduction and replication of RGD-fiber modified adenovirus in primary T cells. PLoS One. 2011; 6:e18091.

22. Kao SH, Su SN, Huang SW, Tsai JJ, Chow LP. Subproteome analysis of novel IgE-binding proteins from Bermuda grass pollen. Proteomics. 2005; 5:3805-13.

23. Reed JC. Drug insight: cancer therapy strategies based on restoration of endogenous cell death mechanisms. Nat Clin Pract Oncol. 2006; 3:388-98.

24. Shinoura N, Yoshida Y, Sadata A, Hanada KI, Yamamoto S, Kirino T, Asai A, Hamada H. Apoptosis by retrovirus- and adenovirus-mediated gene transfer of Fas ligand to glioma cells: implications for gene therapy. Hum Gene Ther. 1998; 9:1983-93. 
25. Reed JC, Pellecchia M. Apoptosis-based therapies for hematologic malignancies. Blood. 2005; 106:408-18.

26. Ng SS, Tsao MS, Nicklee T, Hedley DW. Effects of the epidermal growth factor receptor inhibitor OSI-774, Tarceva, on downstream signaling pathways and apoptosis in human pancreatic adenocarcinoma. Mol Cancer Ther. 2002; 1:777-83.

27. Donegan SE, Naples KM. Epidermal growth factor receptor inhibitors: a novel anticancer therapy. Cancer Pract. 2002; 10:53-6.

28. Juang SH, Wei SJ, Hung YM, Hsu CY, Yang DM, Liu KJ, Chen WS, Yang WK. IFN-beta induces caspase-mediated apoptosis by disrupting mitochondria in human advanced stage colon cancer cell lines. J Interferon Cytokine Res. 2004; 24:231-43

29. Gao H, Gong XC, Chen ZD, Xu XS, Zhang Q, Xu XM. Induction of apoptosis in hormone-resistant human prostate cancer PC3 cells by inactivated Sendai virus. Biomed Environ Sci. 2014; 27:506-14.

30. Johns TG, Mackay IR, Callister KA, Hertzog PJ, Devenish RJ, Linnane AW. Antiproliferative potencies of interferons on melanoma cell lines and xenografts: higher efficacy of interferon beta. J Natl Cancer Inst. 1992; 84:1185-90.

31. Salmon P, Le Cotonnec JY, Galazka A, Abdul-Ahad A, Darragh A. Pharmacokinetics and pharmacodynamics of recombinant human interferon-beta in healthy male volunteers. J Interferon Cytokine Res. 1996; 16:759-64.
32. Einhorn S, Grander D. Why do so many cancer patients fail to respond to interferon therapy? J Interferon Cytokine Res. 1996; 16:275-81.

33. Hickman JA. Apoptosis induced by anticancer drugs. Cancer Metastasis Rev. 1992; 11:121-39.

34. Broker LE, Kruyt FA, Giaccone G. Cell death independent of caspases: a review. Clin Cancer Res. 2005; 11:3155-62.

35. Nakamura K, Ito Y, Kawano Y, Kurozumi K, Kobune M, Tsuda H, Bizen A, Honmou O, Niitsu Y, Hamada H. Antitumor effect of genetically engineered mesenchymal stem cells in a rat glioma model. Gene Ther. 2004; 11: 1155-64.

36. Azizi SA, Stokes D, Augelli BJ, DiGirolamo C, Prockop DJ. Engraftment and migration of human bone marrow stromal cells implanted in the brains of albino rats--similarities to astrocyte grafts. Proc Natl Acad Sci U S A. 1998; 95: 3908-13.

37. Kopen GC, Prockop DJ, Phinney DG. Marrow stromal cells migrate throughout forebrain and cerebellum, and they differentiate into astrocytes after injection into neonatal mouse brains. Proc Natl Acad Sci U S A. 1999; 96:10711-6.

38. Weiss ML, Medicetty S, Bledsoe AR, Rachakatla RS, Choi M, Merchav S, Luo Y, Rao MS, Velagaleti G, Troyer D. Human umbilical cord matrix stem cells: preliminary characterization and effect of transplantation in a rodent model of Parkinson's disease. Stem Cells. 2006; 24:781-92. 